\title{
Evaluation of the optimal dosage of S-1 in adjuvant SOX chemotherapy for gastric cancer
}

\author{
LIN YANG $^{1 *}$, YI YANG ${ }^{2 *}$, QIONG QIN ${ }^{1}$, AIPING ZHOU $^{1}$, JIANJUN ZHAO $^{1}$, JINWAN WANG $^{1}$, \\ CHANG SHU ${ }^{2}$, XINGHUA YUAN ${ }^{1}$ and SONGNIAN HU ${ }^{2}$ \\ ${ }^{1}$ Chinese Academy of Medical Sciences, Cancer Institute and Hospital, Beijing 100021; \\ ${ }^{2}$ Chinese Academy of Sciences, Beijing Institute of Genomics, Beijing 100101, P.R. China
}

Received April 13, 2014; Accepted December 3, 2014

DOI: $10.3892 / \mathrm{ol} .2014 .2821$

\begin{abstract}
Gastric cancer (GC) is the second leading cause of cancer-related mortality worldwide. The usual treatment of GC consists of surgery with additional adjuvant chemotherapy. In the present study, the feasibility and safety of adjuvant S-1 plus oxaliplatin (SOX) chemotherapy for patients with GC and the optimal dosage of S-1 were determined. Eligible patients were randomly assigned to either arm A (30 cases) receiving $70 \mathrm{mg} / \mathrm{m}^{2} \mathrm{~S}-1$ (in two seperate half doses) daily or arm B (30 cases) receiving $80 \mathrm{mg} / \mathrm{m}^{2} \mathrm{~S}-1$ (in two seperate half doses) daily. The S-1 was administered twice daily for 14 days followed by a 7-day rest period for the third week. A total of $130 \mathrm{mg} / \mathrm{m}^{2}$ oxaliplatin was administered on day 1 every 3 weeks for each arm. The cumulative rates of the relative total administration dose of S-1 at $100 \%$ in the 6 th treatment course was $71.4 \%$ [95\% confidence interval (CI), 56.5-90.3\%] in arm A, which was significantly higher than $21.4 \%(95 \% \mathrm{CI}, 10.5-43.6 \%)$ in arm $\mathrm{B}(\mathrm{P}=0.001)$. The most common grade $3 / 4$ toxicities were neutropenia $(19.6 \%)$, thrombocytopenia (19.6\%) and vomiting (16.1\%). Grade 3/4 thrombocytopenia was observed in $7.1 \%$ of patients in arm A and in $32.1 \%$ of patients in arm $\mathrm{B}(\mathrm{P}=0.019)$. With regard to the adverse events induced by $\mathrm{S}-1$ administration, the incidence of diarrhea (3.6 vs. $42.9 \%$; $\mathrm{P}<0.001$ ) was significantly higher in arm B than in arm A, as anticipated. Collectively, adjuvant SOX therapy for GC is feasible and safe, and when
\end{abstract}

Correspondence to: Dr Lin Yang, Chinese Academy of Medical Sciences, Cancer Institute and Hospital, 17 Panjiayuannanli, Beijing 100201, P.R. China

E-mail: yanglin@csco.org.cn

Dr Songnian Hu, Chinese Academy of Sciences, Beijing Institute of Genomics, 1 Beichen West Road, Beijing 100101, P.R. China

E-mail: husn@big.ac.cn

*Contributed equally

Key words: adjuvant chemotherapy, gastric cancer, S-1, oxaliplatin combined with $130 \mathrm{mg} / \mathrm{m}^{2}$ oxaliplatin, $70 \mathrm{mg} / \mathrm{m}^{2} /$ day S-1 appears to the optimal dose.

\section{Introduction}

Gastric cancer (GC) is the second most common cause of cancer-related mortality worldwide and the third most common cancer in China $(1,2)$. The primary treatment for operable GC is surgery. However, recurrence rates are high when using surgery only $(3,4)$. Compared with surgery only, additional adjuvant chemotherapy has shown clinical benefits in treating GC when evaluated by meta-analyses $(5,6)$. Therefore, it is necessary to develop optimal adjuvant chemotherapy regimens to decrease recurrence and improve the quality of life for GC patients following surgical resection.

$\mathrm{S}-1$ is a fourth generation oral fluoropyrimidine, which contains tegafur/gimeracil/oteracil potassium in a molar ratio of 1.0:0.4:1.0. Treatment with S-1 subsequent to surgery has been shown to improve the 5 year overall survival (OS) rate of GC patients from $61.1 \%$ with surgery alone to $71.1 \%$, and the relapse-free survival rate of GC patients in 5 years from 53.1 to $65.4 \%$ (7). However, a subgroup data analysis showed that use of adjuvant chemotherapy with S-1 alone following surgery for patients diagnosed with stage III GC did not result in improved survival (7).

For metastatic or recurrent GC, adjuvant chemotherapy with S-1 plus cisplatin (SP) showed improved results, with a longer progression-free survival (PFS) time and a longer OS time, compared with S-1 alone (8). Compared with cisplatin, oxaliplatin has a more favorable safety profile, including less emetogenic and less nephrotoxic potential. A REAL-2 study revealed that a oxaliplatin-based regimen was just as effective as a cisplatin-based regimen in patients with previously untreated advanced GC (AGC) (9). Additionally, a large randomized phase III study recently reported that $\mathrm{S}-1$ plus oxaliplatin (SOX) showed non-inferiority to SP in PFS and that the treatment was well tolerated, with benefits in terms of outpatient-based treatment in patients with AGC (10).

In our previous study, the SOX regimen with $130 \mathrm{mg} / \mathrm{m}^{2}$ oxaliplatin was found to be effective and safe to use as a first-line chemotherapy in patients with AGC (11). Therefore, the dose of oxaliplatin was fixed at $130 \mathrm{mg} / \mathrm{m}^{2}$ in the current study. A dose of $\mathrm{S}-1$ at $80 \mathrm{mg} / \mathrm{m}^{2}$ (in two seperate half doses) twice daily on days 1 
to 14 every 21 days is widely used for phase II/III studies $(12,13)$. However, in our previous dose-finding study on adjuvant chemotherapy with SOX (130 mg/m² oxaliplatin) for GC, the maximum tolerated dose of $\mathrm{S}-1$ was initially determined to be $70 \mathrm{mg} / \mathrm{m}^{2}$ (14). Grade 3 vomiting was observed as dose-limiting toxicity (DLT) during the first treatment cycle in this study, highlighting the fact that oxaliplatin may play an important role in DLT. Based on these studies, we hypothesized that from the two doses ( $70 \mathrm{vs} .80 \mathrm{mg} / \mathrm{m}^{2} /$ day) there should be an optimal dosage of S-1 when combined with oxaliplatin $\left(130 \mathrm{mg} / \mathrm{m}^{2}\right)$ in adjuvant chemotherapy for GC. The aim of the present study was therefore to evaluate the feasibility and safety of adjuvant SOX chemotherapy for GC patients. In addition, the present study aimed to determine the optimal dosage of S-1 in the SOX combination for GC patients.

\section{Patients and methods}

Patients. A total of 60 eligible patients at the Cancer Institute and Hospital of the Chinese Academy of Medical Sciences (Beijing, China) were recruited for this study according to the following inclusion criteria: Subtotal or total gastrectomy; histologically proven stage II/III [i.e., pathological stage T2N+, T3-T4 and/or N+, according to the American Joint Committee on Cancer tumor-node-metastasis system, 7th edition (15)] GC of the stomach or gastroesophageal junction; age distribution of 20 to 75 years old; an Eastern Cooperative Oncology Group performance status (16) of 0-1; an absolute granulocyte count of $>1,500 / 1$; a platelet count of $>100,000 / 1$; a hemoglobin level of $>90 \mathrm{~g} / \mathrm{l}$; a serum bilirubin level of less than the upper limit of normal (ULN); a normal creatinine level; an alanine transaminase and aspartate transaminase level of $<1.5 \mathrm{xULN}$; and no treatment with chemotherapy prior to the present SOX treatment. Only patients who could swallow tablets were admitted into the study group, and all patients were told to practice medically effective contraception.

Study approval and consent. The present study was approved by the Ethics Committee of the Cancer Institute and Hospital, (Chinese Academy of Medical Sciences, Beijing, China). All patients provided written informed consent.

Treatment schedule. Treatment with SOX therapy was started at 4-8 weeks post-surgery, and repeated for 8 cycles. All eligible patients were randomly assigned to either arm A receiving S-1 at a dose of $70 \mathrm{mg} / \mathrm{m}^{2} /$ day (in two seperate half doses) or arm B receiving S-1 at a dose of $80 \mathrm{mg} / \mathrm{m}^{2} /$ day (in two seperate half doses). S-1 was administered orally twice per day, within half an hour of a meal on days 1 to 14, every 3 weeks (1 cycle). Oxaliplatin was administered intravenously to all patients on day 1 every 3 weeks at a fixed dose of $130 \mathrm{mg} / \mathrm{m}^{2}$. All patients received 5-HT3 antagonists as antiemetics following administration of oxaliplatin. If patients developed grade 4 neutropenia or grade 3/4 thrombocytopenia, or non-hematological toxic effects of above grade 2, the dose of S-1 was reduced by $10 \mathrm{mg} / \mathrm{m}^{2} /$ day, and at the same time, the dose of oxaliplatin was reduced by $25 \%$. If recovery from such toxicities was confirmed at a reduced dose, the administration at the reduced dosage was continued. S-1 and oxaliplatin could be reduced twice, but treatment was discontinued if subsequent reduction was indicated.
In cases of oxaliplatin-related neurological adverse events, S-1 could be continued as monotherapy. Oxaliplatin monotherapy was not allowed if S-1 was discontinued.

Complete blood count and blood chemistry studies were performed weekly. Administration of the two agents would be delayed until adequate hematological recovery (absolute neutrophil count, $\geq 1.5 \times 10^{9} / 1$; platelet count, $\geqq 100 \times 10^{9} / 1$ ) was achieved. Non-hematological toxicities, excluding alopecia, were required to be grade 1 or better prior to initiation of each cycle. If the toxicity failed to recover within 3 weeks after the scheduled day for starting the next cycle, the patients were withdrawn from the study. Therapy was discontinued if there was any evidence of documented recurrence, unacceptable toxicities or refused treatment.

Evaluation. All eligible patients were considered to be assessable for feasibility and safety. Adverse events were graded according to the National Cancer Institute-Common Toxicity Criteria version 4.0 (17). Feasibility was evaluated by the completion status of the protocol treatment and compared between the two arms. The rate of completing $\geq 6$ and 8 cycles of treatment, delayed courses and dose reduction was evaluated and compared in the two arms. The number of patients was calculated at the time when the treatment was stopped or delayed, and when the planned administration dose was reduced. In the two arms, the relative total administration dose in the 6th treatment course (R6) and the 8th treatment course (R8) were calculated as follows: $\mathrm{R} 6=(\mathrm{D} 1+\mathrm{D} 2+\mathrm{D} 3+\mathrm{D} 4+\mathrm{D} 5+\mathrm{D} 6) /(\mathrm{P} 1 \times 6)$ and $\mathrm{R} 8=(\mathrm{D} 1+\mathrm{D} 2+\mathrm{D} 3+\mathrm{D} 4+\mathrm{D} 5+\mathrm{D} 6+\mathrm{D} 7+\mathrm{D} 8) /(\mathrm{P} 1 \times 8)$, where $\mathrm{R}$ is the relative total administration dose, $\mathrm{D}$ is the actual dose in each cycle and P1 is the planned dose in the first cycle. If no treatment was offered in this cycle, the actual dose was set to zero. The cumulative rate of the relative total administration dose in the 6th and 8th treatment courses were also calculated and compared in two arms.

Statistics. Patient characteristics, feasibility and adverse events were analyzed. The differences in the median body surface area and ages between the two arms were evaluated by the Mann-Whitney U test, while other characteristics were evaluated by the $\chi^{2}$ test. The differences in completion status of protocol treatment and adverse events between the two arms were evaluated by the $\chi^{2}$ test. The differences in the relative total administration dose between the two arms were compared by Student's t-test. The cumulative rates of the relative total administration dose of S-1 and oxaliplatin were examined by the Kaplan-Meier method and differences in the two arms were calculated by the log-rank test. Two-sided $\mathrm{P}<0.05$ was used to indicate a statistically significant difference.

\section{Results}

Study population. Between June 2011 and June 2013, 60 patients were recruited to the study. Among these, two patients in arm A and two patients in arm B refused treatment and were excluded from all analyses. Patient disposition throughout the study is shown in Fig. 1. The major reasons for discontinuation of treatment in arm A were documented recurrence (6.7\%), adverse events $(30 \%)$ and refused treatment (20\%). However, notably, 
Table I. Patient characteristics.

\begin{tabular}{|c|c|c|c|c|c|c|}
\hline \multirow[b]{2}{*}{ Characteristics } & \multicolumn{2}{|c|}{$\operatorname{Arm} A(n=30)$} & \multicolumn{2}{|c|}{ Arm B $(n=30)$} & \multicolumn{2}{|c|}{ Total $(\mathrm{n}=60)$} \\
\hline & No. & $\%$ & No. & $\%$ & No. & $\%$ \\
\hline \multicolumn{7}{|l|}{ Age, years } \\
\hline Median & 53.5 & & 52.5 & & 53 & \\
\hline Range & $28-72$ & & $27-67$ & & $27-72$ & \\
\hline \multicolumn{7}{|l|}{$\mathrm{BSA}, \mathrm{m}^{2}$} \\
\hline Median & 1.705 & & 1.705 & & 1.705 & \\
\hline Range & $1.35-2.01$ & & $1.48-1.98$ & & $1.35-2.01$ & \\
\hline \multicolumn{7}{|l|}{ Gender } \\
\hline Male & 24 & 80.0 & 23 & 76.7 & 47 & 78.3 \\
\hline Female & 6 & 20.0 & 7 & 23.3 & 13 & 21.7 \\
\hline \multicolumn{7}{|l|}{ ECOS PS } \\
\hline 0 & 13 & 43.3 & 13 & 43.3 & 26 & 43.3 \\
\hline 1 & 17 & 56.7 & 17 & 56.7 & 34 & 56.7 \\
\hline \multicolumn{7}{|c|}{ Type of gastrectomy } \\
\hline Total & 6 & 20.0 & 5 & 16.7 & 11 & 18.3 \\
\hline Partial & 24 & 80.0 & 25 & 83.3 & 49 & 81.7 \\
\hline \multicolumn{7}{|l|}{ TNM stage } \\
\hline IA & 2 & 6.7 & 2 & 6.7 & 4 & 6.7 \\
\hline II & 9 & 30.0 & 8 & 26.7 & 17 & 28.3 \\
\hline III & 18 & 60.0 & 19 & 63.3 & 37 & 61.7 \\
\hline IV & 1 & 3.3 & 1 & 3.3 & 2 & 3.3 \\
\hline
\end{tabular}

ECOS PS, Eastern Cooperative Oncology Group performance status; BSA, body surface area; TNM, tumor-node-metastasis.

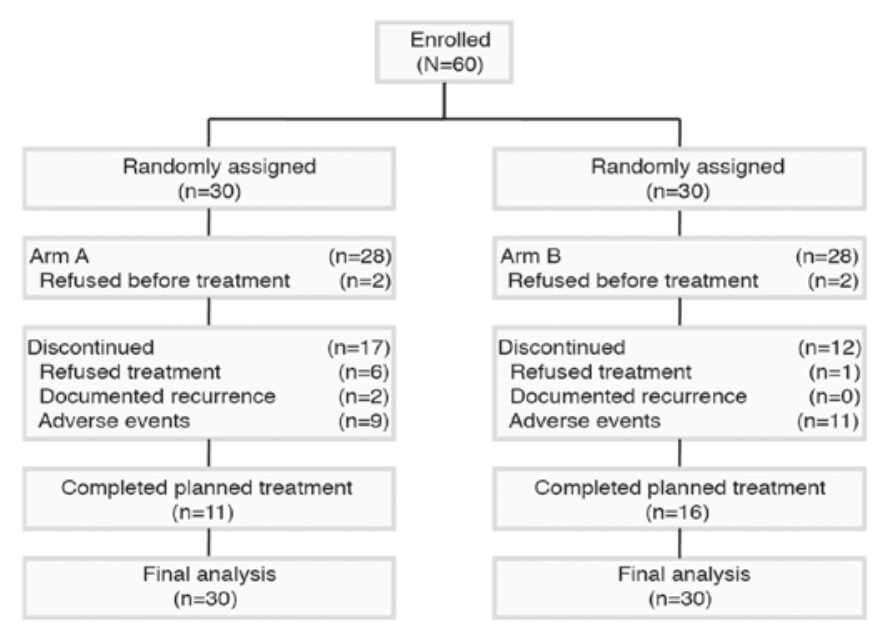

Figure 1. CONSORT (18) diagram showing patient disposition. Arm A, $70 \mathrm{mg} / \mathrm{m}^{2} /$ day S-1; arm B, $80 \mathrm{mg} / \mathrm{m}^{2} /$ day S-1.

four patients refused treatment subsequent to finishing 6 cycles of therapy in arm A. Patient baseline characteristics were well balanced between the two arms (Table I).

Feasibility. In total, $48.2 \%$ of patients completed the planned 8 cycles of treatment and $75 \%$ of patients completed 6 cycles of therapy. A total of $39.3 \%$ of patients in arm A received the full 8 cycles of SOX compared with $57.1 \%$ in arm B [not significant (NS), $\mathrm{P}=0.181$ ] (Table II). However, $82.1 \%$ of patients in arm $A$ received $\geq 6$ cycles of therapy, which was higher the percentage of $67.9 \%$ in arm B (NS, $\mathrm{P}=0.217)$. In total, $91.1 \%$ of patients were observed with delayed courses. Among them, $82.1 \%$ of patients underwent delayed courses in $\operatorname{arm~} \mathrm{A}$, while $100 \%$ were observed in $\operatorname{arm~B~}(\mathrm{P}=0.019)$. In total, the rate of patients with a dose-reduction of S-1 and oxaliplatin was $33.9 \%$ and $42.9 \%$, respectively. The rate of patients with a dose-reduction of $\mathrm{S}-1$ was $14.3 \%$ in $\operatorname{arm} \mathrm{A}$ and $53.6 \%$ in $\operatorname{arm} \mathrm{B}(\mathrm{P}=0.002)$. In the patients with a dose-reduction of oxaliplatin, a rate of $39.3 \%$ was observed in $\operatorname{arm} \mathrm{A}$ and $46.4 \%$ was observed in arm $\mathrm{B}(\mathrm{NS}, \mathrm{P}=0.558)$.

The mean of the relative total administration dose of $\mathrm{S}-1$ in the 6th treatment course was $89.43 \%$ in arm A and $81.36 \%$ in arm $\mathrm{B}(\mathrm{NS}, \mathrm{P}=0.213)$, and the mean of the relative total administration dose of $\mathrm{S}-1$ in the 8 th treatment course in arm A was higher than that in arm B (77.18 vs. $73.07 \%$; NS, $\mathrm{P}=0.551$ ) (Table III). The mean of the relative total administration dose of oxaliplatin in the 6th treatment course was $83.57 \%$ in arm $\mathrm{A}$ and $81.89 \%$ in arm $\mathrm{B}$ (NS, $\mathrm{P}=0.810)$, and the mean of the relative total administration dose of oxaliplatin in the 8th treatment course in arm A was higher that in arm B (66.57 vs. 70.68\%; NS, $\mathrm{P}=0.540$ ) (Table III).

The cumulative rates of the relative total administration dose of S-1 in the 6th treatment course at $100 \%$ was $71.4 \%$ (95\% CI, 56.5-90.3\%) in arm A, which was significantly higher than the $21.4 \%(95 \% \mathrm{CI}, 10.5-43.6 \%)$ in $\operatorname{arm} \mathrm{B}$ 
Table II. Completion status of protocol treatment.

\begin{tabular}{|c|c|c|c|c|c|c|c|}
\hline & \multicolumn{2}{|c|}{ Total $(n=56)$} & \multicolumn{2}{|c|}{ Arm A $(n=28)$} & \multicolumn{2}{|c|}{ Arm B $(n=28)$} & \multirow[b]{2}{*}{ P-value } \\
\hline & No. & $\%$ & No. & $\%$ & No. & $\%$ & \\
\hline Patients received $\geq 6$ cycles of therapy & 42 & 75.0 & 23 & 82.1 & 19 & 67.9 & 0.217 \\
\hline Patients received 8 cycles of therapy & 27 & 48.2 & 11 & 39.3 & 16 & 57.1 & 0.181 \\
\hline Patients with delayed courses & 51 & 91.1 & 23 & 82.1 & 28 & 100.0 & 0.019 \\
\hline \multicolumn{8}{|l|}{ Patients with dose-reduction } \\
\hline S-1 & 19 & 33.9 & 4 & 14.3 & 15 & 53.6 & 0.002 \\
\hline Oxaliplatin & 24 & 42.9 & 11 & 39.3 & 13 & 46.4 & 0.558 \\
\hline
\end{tabular}

Table III. Relative administration dose analysis of S-1 and oxaliplatin.

\begin{tabular}{|c|c|c|c|}
\hline Parameter & $\operatorname{Arm~A~}(\mathrm{n}=28)$ & Arm B (n=28) & P-value \\
\hline $\begin{array}{l}\text { Cumulative rate of relative total administration dose of } \\
\text { S- } 1 \text { at } 100 \% \text { in the } 6 \text { th treatment course, } \%(95 \% \mathrm{CI})\end{array}$ & $\begin{array}{c}71.40 \\
(56.50-90.30)\end{array}$ & $\begin{array}{l}21.40 \\
(10.50-43.60)\end{array}$ & 0.001 \\
\hline Relative total administration dose of S-1 (6th treatment course), $\%$ & & & 0.213 \\
\hline Mean & 89.43 & 81.36 & \\
\hline Standard deviation & 22.08 & 25.70 & \\
\hline $\begin{array}{l}\text { Cumulative rate of relative total administration dose of } \\
\text { S- } 1 \text { at } 100 \% \text { in the } 8 \text { th treatment course, } \%(95 \% \mathrm{CI})\end{array}$ & $\begin{array}{c}32.10 \\
(18.80-55.10)\end{array}$ & $\begin{array}{c}14.30 \\
(5.77-35.40)\end{array}$ & 0.276 \\
\hline Relative total administration dose of $\mathrm{S}-1$ ( 8 th treatment course), \% & & & 0.551 \\
\hline Mean & 77.18 & 73.07 & \\
\hline Standard deviation & 23.74 & 27.36 & \\
\hline $\begin{array}{l}\text { Cumulative rate of relative total administration dose of } \\
\text { OXA at } 100 \% \text { in the } 6 \text { th treatment course, } \%(95 \% \mathrm{CI})\end{array}$ & $\begin{array}{c}46.40 \\
(31.20-69.10)\end{array}$ & $\begin{array}{c}32.10 \\
(18.80-55.10)\end{array}$ & 0.464 \\
\hline Relative total administration dose of OXA (6th treatment course), \% & & & 0.810 \\
\hline Mean & 83.57 & 81.89 & \\
\hline Standard deviation & 24.74 & 27.09 & \\
\hline $\begin{array}{l}\text { Cumulative rate of relative total administration dose of } \\
\text { OXA at } 100 \% \text { in the } 8 \text { th treatment course, } \%(95 \% \mathrm{CI})\end{array}$ & $\begin{array}{c}7.14 \\
(1.88-27.20)\end{array}$ & $\begin{array}{c}14.30 \\
(5.77-35.40)\end{array}$ & 0.230 \\
\hline Relative total administration dose of OXA (8th treatment course), $\%$ & & & 0.540 \\
\hline Mean & 66.57 & 70.68 & \\
\hline Standard deviation & 22.13 & 27.38 & \\
\hline
\end{tabular}

OXA, oxaliplatin; CI, confidence interval.

$(\mathrm{P}=0.001)$ (Fig. 2A; Table III). However, when calculated in the 8th treatment course, the rates were $32.1 \%(95 \%$ $\mathrm{CI}, 18.8-55.1 \%)$ in arm $\mathrm{A}$ and $14.3 \%(95 \% \mathrm{CI}, 5.77-35.4 \%)$ in arm B (NS, $\mathrm{P}=0.276)$ (Fig. 2B; Table III). The cumulative rates of the relative total administration dose of oxaliplatin in the 6th treatment course at $100 \%$ were $46.4 \%$ (95\% CI, $31.2-69.1 \%)$ in arm A and $32.1 \%$ (95\% CI, 18.8-55.1\%) in arm B (NS, P=0.464) (Fig. 2C; Table III). Additionally, when calculated in the 8th treatment course, the rates were 7.14\% (95\% CI, 1.88-27.2\%) in arm A and $14.3 \%$ (95\% CI, $5.77-35.4 \%)$ in arm B (NS, $\mathrm{P}=0.23$ ) (Fig. 2D; Table III).

Adverse events. Drug-related adverse events are listed in Table IV. In total, the most common grade $3 / 4$ hematological toxicities were neutropenia (19.6\%) and thrombocytopenia (19.6\%). A total of $10.7 \%$ of patients in arm A and $28.6 \%$ in arm $\mathrm{B}$ experienced grade $3 / 4$ neutropenia ( $\mathrm{NS}, \mathrm{P}=0.093$ ), while grade $3 / 4$ thrombocytopenia was observed in $7.1 \%$ of patients in arm $\mathrm{A}$ and in $32.1 \%$ of patients in $\operatorname{arm~B}$ $(\mathrm{P}=0.019)$. Grade $1 / 2$ thrombocytopenia was observed at different frequencies in each arm, with 15 patients $(53.6 \%)$ in arm $\mathrm{A}$ and 8 patients (28.6\%) in arm B (NS, $\mathrm{P}=0.057)$. Only one patient in arm B and no patients in arm A developed grade 3 anemia ( $\mathrm{NS}, \mathrm{P}=0.313$ ).

In total, the most common grade $3 / 4$ non-hematological toxicity was vomiting (16.1\%). With regard to the overall incidence of adverse events, hyperpigmentation, asthenia, nausea and neurotoxicity were the most frequent non-hematological 
Table IV. Drug-related adverse events.

\begin{tabular}{|c|c|c|c|c|c|c|c|c|}
\hline \multirow[b]{3}{*}{ Toxicity $^{\mathrm{a}}$} & \multicolumn{2}{|c|}{ Total $(\mathrm{n}=56)$} & \multicolumn{2}{|c|}{$\operatorname{Arm~A~}(n=28)$} & \multicolumn{2}{|c|}{$\operatorname{Arm~B~}(n=28)$} & & \\
\hline & $\mathrm{G} 1 / 2$ & $\mathrm{G} 3 / 4$ & $\mathrm{G} 1 / 2$ & $\mathrm{G} 3 / 4$ & $\mathrm{G} 1 / 2$ & $\mathrm{G} 3 / 4$ & \multicolumn{2}{|c|}{ P-value } \\
\hline & No. $(\%)$ & No. (\%) & No. $(\%)$ & No. $(\%)$ & No. $(\%)$ & No. $(\%)$ & $\mathrm{G} 1 / 2$ & $\mathrm{G} 3 / 4$ \\
\hline Anemia & $20(35.7)$ & $1(1.8)$ & $10(35.7)$ & $0(0.0)$ & $10(35.7)$ & $1(3.6)$ & 1.000 & 0.313 \\
\hline Leukopenia & $39(69.6)$ & $4(7.1)$ & $20(71.4)$ & $1(3.6)$ & $19(67.9)$ & $3(10.7)$ & 0.771 & 0.299 \\
\hline Neutropenia & $32(57.1)$ & $11(19.6)$ & $18(64.3)$ & $3(10.7)$ & $14(50.0)$ & $8(28.6)$ & 0.280 & 0.093 \\
\hline Thrombocytopenia & $23(41.1)$ & $11(19.6)$ & $15(53.6)$ & $2(7.1)$ & $8(28.6)$ & $9(32.1)$ & 0.057 & 0.019 \\
\hline TBIL & $2(3.6)$ & $0(0.0)$ & $2(7.1)$ & $0(0.0)$ & $0(0.0)$ & $0(0.0)$ & 0.150 & - \\
\hline Hyperpigmentation & $33(58.9)$ & $0(0.0)$ & $18(64.3)$ & $0(0.0)$ & $15(53.6)$ & $0(0.0)$ & 0.415 & - \\
\hline Asthenia & $37(66.1)$ & $0(0.0)$ & $17(60.7)$ & $0(0.0)$ & $20(71.4)$ & $0(0.0)$ & 0.397 & - \\
\hline Nausea & 45 (80.4) & $4(7.1)$ & $20(71.4)$ & $2(7.1)$ & $25(89.3)$ & $2(7.1)$ & 0.093 & 1.000 \\
\hline Vomiting & $25(44.6)$ & $9(16.1)$ & $12(42.9)$ & $4(14.3)$ & $13(46.4)$ & $5(17.9)$ & 0.788 & 0.716 \\
\hline Stomatitis & $4(7.1)$ & $0(0.0)$ & $2(7.1)$ & $0(0.0)$ & $2(7.1)$ & $0(0.0)$ & 1.000 & - \\
\hline Diarrhea & $13(23.2)$ & $0(0.0)$ & $1(3.6)$ & $0(0.0)$ & $12(42.9)$ & $0(0.0)$ & $<0.001$ & - \\
\hline Neurotoxicity & $42(75.0)$ & $0(0.0)$ & $22(78.6)$ & $0(0.0)$ & $20(71.4)$ & $0(0.0)$ & 0.537 & - \\
\hline Hand-foot syndrome & $0(0.0)$ & $0(0.0)$ & $0(0.0)$ & $0(0.0)$ & $0(0.0)$ & $0(0.0)$ & - & - \\
\hline ALT elevation & $13(23.2)$ & $0(0.0)$ & $6(21.4)$ & $0(0.0)$ & $7(25.0)$ & $0(0.0)$ & 0.752 & - \\
\hline
\end{tabular}

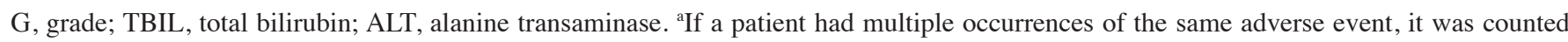
with the highest grade.
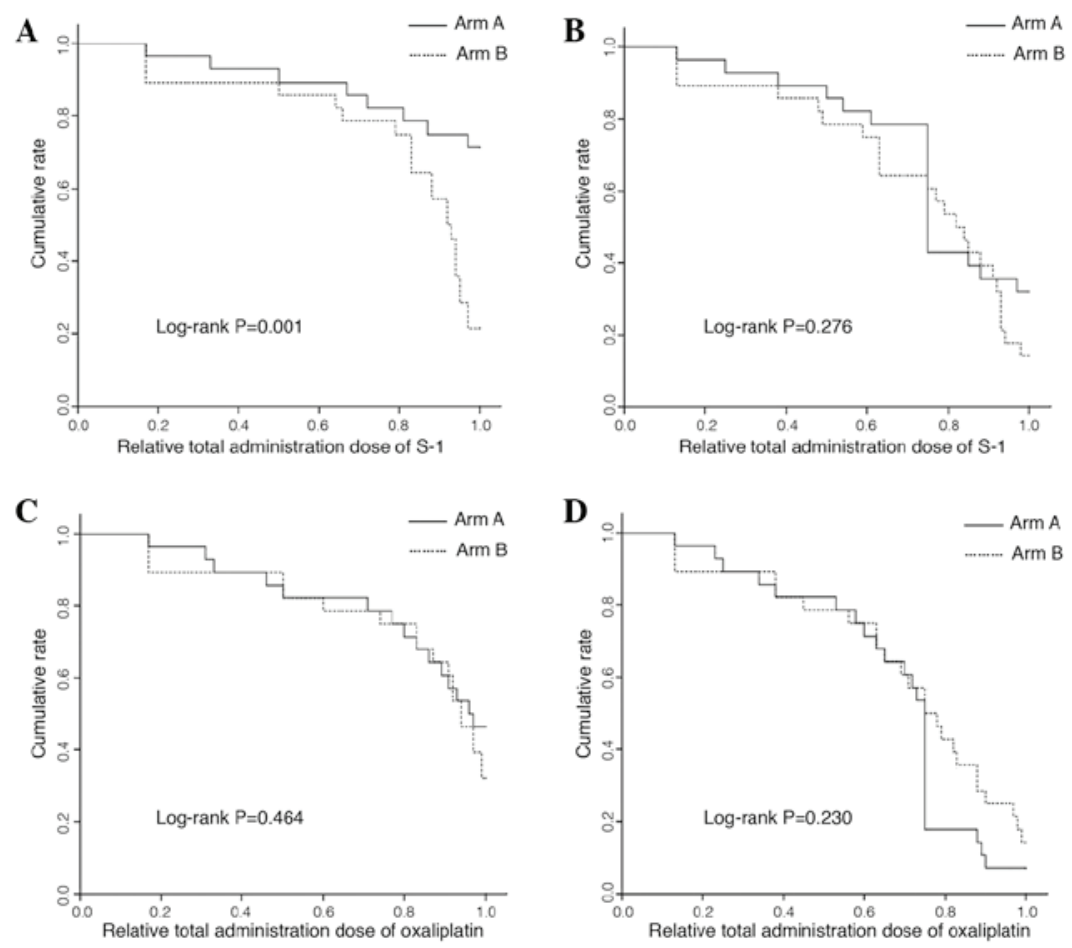

Figure 2. Kaplan-Meier estimation of cumulative rate of relative total administration dose among 56 gastric cancer patients. (A) Cumulative rate of relative total administration dose of S-1 in the 6th treatment course in each arm. (B) Cumulative rate of relative total administration dose of S-1 in the 8th treatment course in each arm. (C) Cumulative rate of relative total administration dose of oxaliplatin in the 6th treatment course in each arm. (D) Cumulative rate of relative total administration dose of oxaliplatin in the 8th treatment course in each arm.

toxicities in each arm. Nausea, with the highest rates of grade $1 / 2$ non-hematological toxicity, was observed in $71.4 \%$ of patients in arm A and $89.3 \%$ in arm B (NS, $\mathrm{P}=0.093)$. With regard to the adverse events induced by $\mathrm{S}-1$ administration, the incidence of diarrhea ( 3.6 vs. $42.9 \%$; $\mathrm{P}<0.001$ ) was significantly higher in arm B compared with arm A, as anticipated. 
Thrombocytopenia was the most frequent reason for dose reduction and cycle delay in each arm. Among the non-hematological toxicities, vomiting in arm A and diarrhea in arm B were the most common reasons for dose reduction and cycle delay.

\section{Discussion}

The role of post-operative adjuvant chemotherapy following curative D2 gastrectomy has long been debated. Multiple randomized, controlled studies have evaluated the role of post-operative adjuvant chemotherapy for GC (19-21), however, as a result of population and regimen heterogeneity, no consensus has been reached with regard to the chemotherapeutic regimen, schedule or duration of adjuvant chemotherapy. The results of the G-SOX study reflected the efficacy and safety of SOX in patients with AGC (12). Therefore, SOX is considered to be a candidate for an experimental arm in the next adjuvant chemotherapy trial.

In the present study, $48.2 \%$ of patients completed 8 cycles as planned, however, $75 \%$ of patients received $>6$ cycles of treatment. SOX has shown more advantages compared with the SP regimen in adjuvant chemotherapy for GC patients, since $22.6 \%$ completed 5 cycles as planned in the CCOG0703 study (22) and $60.8 \%$ completed 6 cycles as planned in the study by Kang et al (23). In the present study, the treatments were generally well tolerated. The most frequently observed grade $3 / 4$ toxicities were neutropenia (19.6\%), thrombocytopenia $(19.6 \%)$ and vomiting $(16.1 \%)$.

To the best of our knowledge, this is the first randomized feasibility study comparing two common doses of S-1 in combination with oxaliplatin in GC patients following curative surgery. From the viewpoint of the completion status of the protocol treatment, the completion rate was not significantly different, however, significantly less delay to the course was observed in arm A (91.1 vs. 82.1\%; $\mathrm{P}=0.019)$. Furthermore, less grade $3 / 4$ neutropenia (19.6 vs. $10.7 \% ; \mathrm{P}=0.093)$ and thrombocytopenia (grade $1 / 2,41.1$ vs. $53.6 \%$; NS, $\mathrm{P}=0.057$; and grade $3 / 4,19.6$ vs. $7.1 \% ; \mathrm{P}=0.019$ ) occurred in arm $\mathrm{A}$, which may have contributed to the less delay to the course. With regard to the adverse events induced by S-1 administration, the incidence of diarrhea (3.6 vs. $42.9 \%$; $\mathrm{P}<0.001$ ) was significantly higher in arm B than in arm A, as anticipated.

The rate of discontinued cases was higher in arm A than in arm B, however, notably, four patients refused treatment subsequent to finishing 6 cycles of therapy in arm A for grade 1 asthenia. The cumulative rate of the relative total administration dose of $\mathrm{S}-1$ in the 6 th treatment course at $100 \%$ was $71.4 \%$ (95\% CI, 56.5-90.3\%) in arm A, which was significantly higher than the $21.4 \%$ (95\% CI, 10.5-43.6\%) in arm $\mathrm{B}(\mathrm{P}=0.001)$. Additionally, when calculated in the 8 th treatment course, the rate was $32.1 \%$ (95\% CI, 18.8-55.1\%) in $\operatorname{arm} \mathrm{A}$, which was higher than the $14.3 \%$ (95\% CI: $5.77-35.4 \%)$ in arm $\mathrm{B}(\mathrm{NS}, \mathrm{P}=0.276)$. The relative total administration dose of $\mathrm{S}-1$ in the 6 th treatment course and the 8th treatment course were higher in arm A than arm B. However, no significant difference in the administration dose of oxaliplatin was found between the two arms.

These results suggested that a regimen using S-1 at a dose of $70 \mathrm{mg} / \mathrm{m}^{2}$ twice daily for 14 days followed by a 7-day rest period is more acceptable compared with the regimen of S-1 at $80 \mathrm{mg} / \mathrm{m}^{2}$ twice daily for 14 days, when combined with oxaliplatin at $130 \mathrm{mg} / \mathrm{m}^{2}$ on day 1 every 3 weeks. Owing to the lack of a survival analysis and the small sample size in the current study, the potential to reduce relapse following treatment in GC patients post-surgery should be carefully examined in the future. Patients in the present study will continue to be observed for recurrence and survival.

\section{Acknowledgements}

S-1 was kindly provided by Taiho Parmaceutical Company (Princeton, NJ, USA).

\section{References}

1. Zhao P, Dai M, Chen W and Li N: Cancer trends in China. Jpn J Clin Oncol 40: 281-285, 2010.

2. Parkin DM, Bray F, Ferlay J and Pisani P: Global cancer statistics, 2002. CA Cancer J Clin 55: 74-108, 2005.

3. Gunderson LL: Gastric cancer - patterns of relapse after surgical resection. Semin Radiat Oncol 12: 150-161, 2002.

4. Gallo A and Cha C: Updates on esophageal and gastric cancers. World J Gastroenterol 12: 3237-3242, 2006.

5. Mari E, Floriani I, Tinazzi A, et al: Efficacy of adjuvant chemotherapy after curative resection for gastric cancer: a meta-analysis of published randomised trials. A study of the GISCAD (Gruppo Italiano per lo Studio dei Carcinomi dell'Apparato Digerente). Ann Oncol 11: 837-843, 2000.

6. Earle CC and Maroun JA: Adjuvant chemotherapy after curative resection for gastric cancer in non-Asian patients: revisiting a meta-analysis of randomised trials. Eur J Cancer 35: 1059-1064, 1999.

7. Sasako M, Sakuramoto S, Katai H, et al: Five-year outcomes of a randomized phase III trial comparing adjuvant chemotherapy with S-1 versus surgery alone in stage II or III gastric cancer. J Clin Oncol 29: 4387-4393, 2011.

8. Koizumi W, Narahara H, Hara T, et al: S-1 plus cisplatin versus S-1 alone for first-line treatment of advanced gastric cancer (SPIRITS trial): a phase III trial. Lancet Oncol 9: 215-221, 2008.

9. Cunningham D, Starling N, Rao S, et al: Upper Gastrointestinal Clinical Studies Group of the National Cancer Research Institute of the United Kingdom: Capecitabine and oxaliplatin for advanced esophagogastric cancer. N Engl J Med 358: 36-46, 2008.

10. Higuchi K, Koizumi W, Yamada Y, et al: Randomized phase III study of S-1 plus oxaliplatin versus S-1 plus cisplatin for first-line treatment of advanced gastric cancer. J Clin Oncol 31 (Suppl 4): astr 60, 2013

11. Yang L, Song Y, Zhou AP, et al: A phase II trial of oxaliplatin plus S-1 as a first-line chemotherapy for patients with advanced gastric cancer. Chin Med J (Engl) 126: 3470-3474. 2013.

12. Koizumi W, Takiuchi H, Yamada Y, et al: Phase II study of oxaliplatin plus S-1 as first-line treatment for advanced gastric cancer (G-SOX study). Ann Oncol 21: 1001-1005, 2010.

13. Narahara $\mathrm{H}$, Iishi $\mathrm{H}$, Imamura $\mathrm{H}$, et al: Randomized phase III study comparing the efficacy and safety of irinotecan plus S-1 with S-1 alone as first-line treatment for advanced gastric cancer (study GC0301/TOP-002). Gastric Cancer 14: 72-80, 2011.

14. Yang L, Yang Y, Qin Q, et al: Dose-finding study on adjuvant chemotherapy with S-1 plus oxaliplatin for gastric cancer. Mol Clin Oncol 2: 93-98, 2014.

15. Edge S, Byrd DR, Compton CC, et al (eds): AJCC Cancer Staging Manual. 7th edition. Springer, New York, NY, 2009.

16. Oken MM, Creech RH, Tormey DC, et al: Toxicity and response criteria of the Eastern Cooperative Oncology Group. Am J Clin Oncol 5: 649-655, 1982.

17. National Cancer Institute: Common Terminology Criteria for Adverse Events [v.4.03]. http://evs.nci.nih.gov/ftp1/CTCAE/CTCAE_4.03_201006-14_QuickReference_5x7.pdf. Accessed June 11, 2011.

18. Schulz KF, Altman DG and Moher D; CONSORT Group: CONSORT 2010 statement: updated guidelines for reporting parallel group randomised trials. PLoS Med 7: e1000251, 2014.

19. Nakajima T, Nashimoto A, Kitamura M, et al: Adjuvant mitomycin and fluorouracil followed by oral uracil plus tegafur in serosa-negative gastric cancer: a randomised trial. Gastric Cancer Surgical Study Group. Lancet 354: 273-277, 1999. 
20. Nakajima T, Takahashi T, Takagi K, Kuno K and Kajitani T: Comparison of 5-fluorouracil with ftorafur in adjuvant chemotherapies with combined inductive and maintenance therapies for gastric cancer. J Clin Oncol 2: 1366-1371, 1984.

21. Nashimoto A, Nakajima T, Furukawa H, et al: Gastric Cancer Surgical Study Group, Japan Clinical Oncology Group: Randomized trial of adjuvant chemotherapy with mitomycin, Fluorouracil, and Cytosine arabinoside followed by oral Fluorouracil in serosa-negative gastric cancer: Japan Clinical Oncology Group 9206-1. J Clin Oncol 21: 2282-2287, 2003.
22. Kodera Y, Ishiyama A, Yoshikawa T, et al: Chubu Clinical Cancer Group: A feasibility study of postoperative chemotherapy with S-1 and cisplatin (CDDP) for gastric carcinoma (CCOG0703). Gastric Cancer 13: 197-203, 2010.

23. Kang BW, Kim JG, Chae YS, et al: Pilot study of adjuvant chemotherapy with 3-week combination of S-1 and cisplatin for patients with stage II-IV (M0) gastric cancer. Invest New Drugs 30: 1671-1675, 2012. 